\title{
Diente más afectado por la celulitis facial de origen odontogénico en niños venezolanos.
}

\section{Resumen:}

Objetivo: Determinar la prevalencia de celulitis facial de origen odontogénica e identificar el diente más afectado en niños con diagnóstico de celulitis facial hospitalizados en el Servicio de Infectología Pediátrica del Hospital Universitario de Caracas en el periodo 2006- 2014. Materiales y métodos: se realizó un estudio documental, descriptivo y retrospectivo en niños entre 1-6 años de edad hospitalizados con diagnóstico de celulitis facial odontogénica en el Servicio de Infectología Pediátrica del Hospital Universitario de Caracas en el periodo 2006-2014. Se tomó una muestra no probabilística a conveniencia, la cual quedó conformada por 310 niños quienes cumplieron los criterios de inclusión de la investigación. Los datos se obtuvieron a partir de las historias clínicas de dicho servicio. Se realizó un análisis des-
Gabriel Zambrano

Rosa Rondón ${ }^{2}$

María Elena Guerra. ${ }^{3}$

\section{Artigos Originais}

criptivo, epidemiológico y comparativo/ analítico para cumplir con los objetivos de la investigación. Resultados: la prevalencia de celulitis facial de origen odontogénico fue de $5,27 \%$ con respecto a la muestra total. El segundo molar primario fue el diente más afectado con una frecuencia del 49,5\%. CONCLUSIÓN: la prevalencia de celulitis facial de origen odontogénico en comparación con otras infecciones que requieren hospitalización fue baja, el género masculino fue el más afectado, el rango de edad más involucrado con la entidad se ubicó entre los 4 y 6 años. Los dientes primarios fueron los más afectados y el diente más asociado con esta afección fue el segundo molar primario.

Palabras clave: Celulitis facial odontogénica, niños, dentición primaria, segundo molar primario.

\section{Dente mais afetado pela celulite facial de origem odontogênico em crianças venezuelanas.}

\section{Resumo:}

Objetivo: Determinar a prevalência da celulite fa- cial de origem odontogênica e identificar o dente mais afetado em crianças com diagnóstico de celulite facial internadas no Serviço de Infectologia Pe-

\footnotetext{
1. Especialista en Odontología Infantil. MSc. en Odontología .Profesor Instructor del Centro de Atención a Pacientes con Enfermedades Infectocon tagiosas "Dra. Elsa La Corte". Facultad de Odontología Universidad Central de Venezuela.

2. Residente de la Maestría en Odontología. Universidad Central de Venezuela.

3. Especialista en Odontología Infantil. Profesora Titular del Centro de Atención a Pacientes con Enfermedades Infectocontagiosas "Dra. Elsa La Corte" Facultad de Odontología Universidad Central de Venezuela.
} 
diátrica do Hospital Universitário de Caracas, no período 2006- 2014. Materiais e métodos: Foi realizada uma estudo documental, descritivo e retrospectivo das crianças entre 1-6 anos de idade internadas com diagnóstico de celulite facial odontogênica no Serviço de Infectologia Pediátrica do Hospital Universitário de Caracas, no período 2006-2014. Foi selecioada uma amostra não probabilística, de conveniencia, foi composta por 310 crianças que preencheram os critérios de inclusão da pesquisa. Os dados foram obtidos a partir dos registros médicos do referido serviço. Foi realizada uma análise descritiva, epidemiológica e comparativa/analítica para atender os objetivos da pesquisa. Resul- tados: A prevalência da celulite facial de origem odontogênica foi de 5,27\% em relação ao total da amostra. O segundo molar decíduo foi o mais afetado, com uma frequência de $49,5 \%$. Conclusão: A prevalência da celulite facial de origem odontogênica comparação com outras infecções que necessitem de hospitalização foi baixa.O sexo masculino foi o mais afetado, a faixa etária mais acometida foi entre 4 e 6 anos. Os dentes decíduos foram os mais afetados e o dente mais associada com esta condição foi o segundo molar decíduo.

Palavras-chave: Celulite odontogênica facial, crianças, dentes decíduos, segundo molar decíduo.

Original Articles

\section{Tooth most affected by odontogenic facial cellulitis in venezuelan children.}

\section{Summary:}

Objective: To determine the prevalence of odontogenic facial cellulitis and identify the most affected tooth in children diagnosed with facial cellulitis hospitalized in the Department of Pediatric Infectious Diseases University Hospital of Caracas in the period 2006-2014. Materials and methods: documentary, descriptive and retrospective study of children between 1-6 years of age hospitalized with a diagnosis of odontogenic facial cellulitis in the Department of Pediatric Infectious Diseases University Hospital of Caracas in the period 2006-2014. A nonrandom convenience sample, which was composed of 310 children who met the inclusion criteria of the investigation was taken. The data were obtained from the medical records of the service.
An analytical, descriptive, epidemiological and comparative analysis to meet the objectives of the research was conducted. Results: The prevalence of facial cellulitis of odontogenic origin was 5.27\% compared to the total sample. The second primary molar was the most affected with a frequency of $49.5 \%$ tooth. Conclusion: The prevalence of facial cellulitis of odontogenic origin compared with other infections requiring hospitalization was low, male gender was the most affected, the age group most involved with the entity was between 4 and 6 years. Primary teeth were the most affected and the tooth most associated with this condition was the second primary molar.

Keywords: odontogenic facial cellulitis, children, primary teeth, second primary molar. 


\section{Introducción:}

La celulitis facial de origen odontogénico constituye un proceso inflamatorio agudo, sin tendencia a la limitación, que abarca varios espacios aponeuróticos de la cara y que se encuentra condicionada por dos factores: la extraordinaria virulencia del microorganismo que la produce y el estado decadente del hospedero que la padece. En relación con el portador, se le da especial atención a factores locales como abscesos de origen endo-periodontales generalmente asociados a caries no tratadas o a tratamientos odontológicos defectuosos. ${ }^{1}$

En la población pediátrica, la celulitis facial se observa con frecuencia en las salas de emergencia hospitalarias. Los pacientes que presentan celulitis facial pueden evidenciar signos de deshidratación, una respuesta deteriorada de su sistema nervioso central, obstrucción de las vías aéreas y compromiso sistémico. ${ }^{2}$

Es importante destacar que las enfermedades infecciosas continúan siendo uno de los problemas más importantes de salud pública a nivel mundial y la región Latinoamericana no escapa de esta situación. ${ }^{3}$

Las infecciones odontogénicas son frecuentes en la población pediátrica, en especial en niños menores de 6 años. En este sentido, la celulitis facial ha tenido un aumento en su prevalencia en las últimas décadas. La determinación del sitio etiológico primario y los microorganismos responsables de la infección puede ser difícil, debido a la proximidad íntima de la piel, dientes, glándulas salivales, senos y conducto auditivo. La flora microbiana y el sitio de origen de una infección pueden variar y deben determinarse para que el régimen del tratamiento más eficaz pueda comenzarse. La celulitis facial odontogénica en niños es similar a la del adulto, aunque en el paciente pediátrico se tendrán consideraciones especiales ya que cursa con una evolución aguda por la facilidad de diseminarse a planos profundos con mayor celeridad. ${ }^{4}$

En el ámbito mundial, la prevalencia de la celulitis facial de origen odontogénico en la pobla- ción infantil va en ascenso, en especial en aquellos países en vías de desarrollo. En este sentido, Grandas A y Velásquez S, en el año 2012 en una investigación llevada a cabo en Colombia reportan una prevalencia de esta entidad de $37,45 \%{ }^{5}$

En Venezuela, a pesar de que se han implementado programas de prevención de caries y de salud bucal, se siguen reportando casos de celulitis facial de origen odontogénico, en especial en niños de bajo estrato social, debido al difícil acceso a la salud odontológica y a la falta de conocimiento en este sentido por parte de sus padres y representantes. En nuestro país no se encontraron trabajos sobre prevalencia de la celulitis facial de origen odontogénico en la población pediátrica, ni se identifica el diente más afectado asociado con esta entidad, siendo pocos los estudios que se han realizado en relación a este tema. Consideramos que es de sumo interés conocer las cifras actuales para así establecer medidas preventivas en las poblaciones afectadas, incentivar la incorporación del Odontopediatra en el trabajo transdisciplinario entre el pediatra y la madre o el cuidador, para de esta forma contribuir con el mejoramiento de la salud pública y de la calidad de vida de los niños venezolanos.

\section{Objetivo:}

Determinar la prevalencia de celulitis facial de origen odontogénica e identificar el diente más afectado en niños con diagnóstico de celulitis facial hospitalizados en el Servicio de Infectología Pediátrica del Hospital Universitario de Caracas en el periodo 2006- 2014.

\section{Materiales y métodos:}

En la presente investigación se consideraron dos aspectos fundamentales para la protección de los derechos y del bienestar de los sujetos participantes: el primero es el relacionado con el consentimiento y la permisología por parte del Jefe del Servicio de Infectología Pediátrica del Hospital Universitario de Caracas para el procedimiento de recolección de datos inherentes al estudio, el segundo y más importante es el concerniente a la 
privacidad, intimidad, confidencialidad y anonimato de la información recolectada para la investigación en base a la Declaración de Helsinki respetando los principios éticos de la investigación científica en seres humanos. ${ }^{6}$ En este sentido, el proyecto de investigación fue sometido a la consideración del Comité de Bioética de la Facultad de Odontología de la Universidad Central de Venezuela, la cual otorgó el Aval bajo el número: CB-OP-013-2015.

Se realizó un estudio combinado, es decir: un estudio documental, un estudio epidemiológico de prevalencia y además se realizó un estudio cuantitativo no experimental, retrospectivo, transversal y analítico en niños entre 1-6 años de edad, hospitalizados con diagnóstico de celulitis facial de origen odontogénico en el Servicio de Infectología Pediátrica del Hospital Universitario de Caracas en el periodo 2006-2014. Para ello, se tomó una muestra no probabilística de conveniencia donde la elección de los participantes dependió de su pertinencia en relación al objetivo planteado.

Criterios de inclusión: niños aparentemente sanos, con edades comprendidas entre 1 y 6 años que fueron hospitalizados en el Servicio de Infectología Pediátrica del Hospital Universitario de Caracas con diagnóstico de celulitis facial de origen odontogénico entre Enero de 2006 a Diciembre de 2014.

Criterios de exclusión: Niños con celulitis facial de origen no odontogénico, niños con patología sistémica de base y/o niños con alteraciones congénitas en el desarrollo de la dentición.

Así, la muestra estuvo constituida por los 310 niños que fueron hospitalizados por celulitis facial de origen odontogénico en el Servicio de Infectología Pediátrica del Hospital Universitario de Caracas entre Enero de 2006 a Diciembre de 2014 que cumplieron con los criterios de inclusión/exclusión del estudio.

Se realizó la recolección de los datos del libro de control de ingresos de hospitalización del Servicio de Infectología Pediátrica del Hospital Universitario de Caracas y de las historias clínicas, empleando un instrumento de recolección de datos elaborado por los investigadores, de todos los pacientes con diagnóstico de celulitis facial odontogénica y que cumplieron con los criterios de inclusión.

Los datos se recolectaron en un instrumento ad hoc que fue creado especialmente para este estudio.

Todos los datos vaciados en la base de datos del paquete estadístico Statistical Package for the Scientific Sciences (SPSS) versión 21.0.

Se realizó un análisis descriptivo, con la finalidad de caracterizar la muestra, basado en frecuencias y porcentajes. Un análisis epidemiológico, con la finalidad de determinar la prevalencia de la de celulitis facial de origen odontogénico en la población estudiada en este centro, basado en la prueba estadística de Chi Cuadrado de Independencia entre variables y un análisis comparativo/ analítico utilizando las variables: edad, género, grupo dental y diente más afectado.

\section{Resultados:}

Se encontró un total de 5882 pacientes que fueron hospitalizados en el Servicio de Infectología Pediátrica del Hospital Universitario de Caracas en edades comprendidas entre 1 a 6 años, en el periodo estudiado, de los cuales 723 presentaban diagnóstico de celulitis facial, sin embargo solo 310 cumplían los criterios de inclusión de la muestra. En este sentido, la prevalencia de celulitis facial de origen odontogénico fue de 5,27\% con respecto a la muestra total. Lo que puede indicar que de cada 100 niños, cinco (5) pueden presentar celulitis facial de origen odontogénico. Sin embargo, en relación con la prevalencia de celulitis facial de origen odontogénico en base al total de la población con diagnóstico de celulitis facial, se determinó que la misma era de 42,87\%, pudiendo indicar que de cada 100 niños hospitalizados en este Servicio con diagnóstico de celulitis facial, 43 son de origen odontogénico.

En la Tabla 1 se puede observar la Tasa de Prevalencia por año de Celulitis Facial de origen 


\begin{tabular}{|cccc|} 
Año & $\begin{array}{c}\text { Población } \\
\text { atendida }\end{array}$ & $\begin{array}{c}\text { No de casos } \\
\text { Prevalencia } \\
\%\end{array}$ \\
\hline 2006 & 557 & 47 & 8,438 \\
\hline 2007 & 599 & 29 & 4,841 \\
\hline 2008 & 672 & 30 & 4,464 \\
\hline 2009 & 502 & 40 & 7,968 \\
\hline 2010 & 662 & 39 & 5,891 \\
\hline 2011 & 549 & 28 & 5,100 \\
\hline 2012 & 674 & 34 & 5,045 \\
\hline 2013 & 692 & 26 & 3,757 \\
\hline 2014 & 975 & 37 & 3,795 \\
\hline
\end{tabular}

Tabla 1. Tasa de prevalencia de celulitis facial de origen odontogénico por año en pacientes entre 1 a 6 años de edad hospitalizados en el Servicio de Infectología Pediátrica del Hospital Universitario de Caracas en el periodo 2006-2014.

Odontogénico en pacientes entre 1 a 6 años de edad hospitalizados en el Servicio de Infectología Pediátrica del Hospital Universitario de Caracas entre Enero 2006 - Diciembre del 2014.

De la población de niños hospitalizados en el Servicio de Infectología Pediátrica en edades comprendidas entre 1 a 6 años, en el periodo Enero 2006 - Diciembre de 2014, la prevalencia de celulitis facial de origen odontogénico presentó variaciones, registrándose para el año 2006 una mayor prevalencia $(8,4$ por cada 100 pacientes), que luego fue disminuyendo considerablemente en los años 2007 y 2008, repuntando para el año 2009 (7,9 por cada 100 pacientes) y posteriormente ir disminuyendo considerablemente y mante-

\begin{tabular}{|c|c|c|c|c|c|c|}
\hline \multicolumn{7}{|c|}{ Rango de Edades } \\
\hline $\begin{array}{c}\text { Celulitis } \\
\text { Facial } \\
\text { Odontogénica }\end{array}$ & $\mathbf{N}$ & $\%$ & $\mathbf{N}$ & $\%$ & $\mathbf{N}$ & $\%$ \\
\hline Presencia & 107 & 3,1 & 203 & 8,5 & 310 & 5,2 \\
\hline Ausencia & 3394 & 96,9 & 2178 & 91,5 & 5575 & 94,8 \\
\hline Total & 3501 & 100 & 2381 & 100 & 5882 & 100 \\
\hline
\end{tabular}

Tabla 3. Prevalencia de celulitis facial de origen odontogénico en pacientes hospitalizados en el Servicio de Infectología Pediátrica del Hospital Universitario de Caracas entre enero 2006 - diciembre del 2014 de acuerdo al rango de edad.

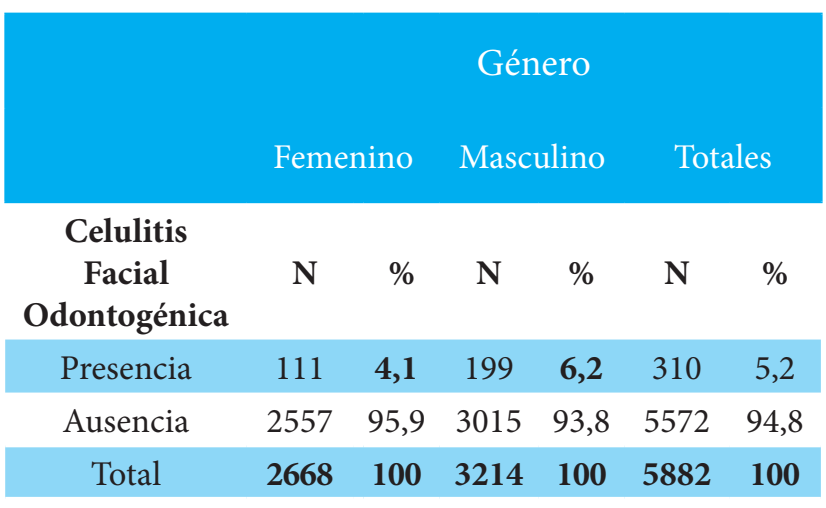

Tabla 2. Prevalencia de celulitis facial de origen odontogénico en pacientes entre 1 a 6 años de edad hospitalizados en el Servicio de Infectología Pediátrica del Hospital Universitario de Caracas entre enero 2006 - diciembre del 2014 de acuerdo al género.

niéndose estable (3,7 por cada 100 pacientes) en los años 2013 y 2014.

La prevalencia de celulitis facial de origen odontogénico en la población estudiada de acuerdo al género se puede apreciar en la Tabla 2.

De la población de niños considerados en esta investigación se observa que hay una mayor prevalencia del género masculino (6 por cada 100) respecto al femenino (4 por cada 100), sin embargo al aplicar la prueba estadística de Chi-Cuadrado no se encuentra evidencia significativa $p=0,063$ ( $p$-valor $>0,05)$ que indique que el género pueda estar relacionado con la presencia de la celulitis facial de origen odontogénico.

En la Tabla 3 se denota la prevalencia de celulitis facial de origen odontogénico en la población estudiada, la cual se dividió en dos grupos etarios: 1-3 años y de 4-6 años.

En la población estudiada, se observa que existe una mayor prevalencia de presentar celulitis facial de origen odontogénico en niños cuya edad está comprendida entre los 4 y 6 años (8,5\%), en este sentido y al efectuar la prueba estadística de Chi-Cuadrado de independencia, se encontró que hay relevancia estadísticamente significativa $\mathrm{p}=$ 0,033 ( $p$-valor $<0,05$ ), lo que indica que la edad es un factor que puede estar relacionado con la presencia de la celulitis facial de origen odontogénico. 


\begin{tabular}{|ccc}
$\begin{array}{c}\text { Grupo dental } \\
\text { afectado }\end{array}$ & Frecuencia & Porcentaje (\%) \\
\hline Primario & 238 & $\mathbf{7 6 , 8}$ \\
\hline Permanente & 21 & 6,7 \\
\hline Ambos & 51 & 16,5 \\
\hline Total & $\mathbf{3 1 0}$ & $\mathbf{1 0 0}$ \\
\hline
\end{tabular}

Tabla 4. Distribución de frecuencia según el grupo dental afectado de los niños que presentaron celulitis facial de origen odontogénico entre 1 a 6 años de edad hospitalizados en el Servicio de Infectología Pediátrica del Hospital Universitario de Caracas entre enero de 2006 a diciembre de 2014.

El grupo dental más afectado en este estudio fue con mayor prevalencia el grupo de dientes primarios en un $76,8 \%$ de los casos, mientras que los dientes permanentes sólo se vieron involucrados en un 6,7\% de los pacientes. Sin embargo, es importante destacar que en 51 pacientes se encontraron afectados dientes primarios y permanentes contiguos, lo que representa el 16,5\% del total. Estos resultados se aprecian en la Tabla 4. Por su parte, en la Tabla 5 se aprecian los resultados obtenidos con respecto al diente más afectado en este estudio. El segundo molar primario fue el diente más afectado con una frecuencia del $49,5 \%$, seguido inmediatamente por el primer molar primario con un $22,3 \%$ de la muestra total. La afección del segundo molar primario y del primer molar permanente ocupó el tercer lugar en frecuencia con un $16,6 \%$. En cuanto al primer molar permanente se ubicó en el cuarto lugar con una frecuencia del 5,5\%.

En este estudio, se evaluó a nivel estadístico si existía relación entre el diente afectado con la celulitis facial de origen odontogénico, aplicando el estadístico Chi Cuadrado y se obtuvo un valor de significancia $\mathrm{p}=0,316$ ( $\mathrm{p}$-valor $>0,05$ ), dando como resultado que no existe relación significativa entre el diente afectado y la presencia de celulitis facial de origen odontogénico en los niños de 1 a 6 años de edad considerados en esta investigación. Sin embargo, al aplicar el estadístico de Correlación de Pearson se encontró que existe relación estadísticamente significativa entre la presencia de celulitis facial de origen odontogénica y la afección en dientes primarios (incisivos, caninos y molares primarios asociados con la en- tidad) al encontrar un $p=0,000$ ( $p$-valor $>0,05)$ y un coeficiente de relación fuerte del $94,5 \%$.

\section{Discusión:}

La celulitis facial suele observarse con frecuencia en niños hospitalizados y puede ser clasificada de acuerdo a la fuente de la infección en odontogénica y no odontogénica. Las celulitis faciales odontogénicas son infecciones que se derivan de los dientes y/o de sus tejidos de soporte periodontales. Constituyen aproximadamente el 50\% del total de las celulitis faciales en pacientes pediátricos. $^{7}$

\begin{tabular}{|c|c|c|}
\hline Diente afectado & Frecuencia & Porcentaje (\%) \\
\hline $\begin{array}{l}\text { Incisivo central } \\
\text { primario }\end{array}$ & 7 & 2,3 \\
\hline $\begin{array}{l}\text { Incisivo lateral } \\
\text { primario }\end{array}$ & 4 & 1,3 \\
\hline Canino primario & 1 & 0,3 \\
\hline $\begin{array}{l}\text { Primer molar } \\
\text { primario }\end{array}$ & 69 & 22,3 \\
\hline $\begin{array}{l}\text { Segundo molar } \\
\text { primario }\end{array}$ & 153 & 49,5 \\
\hline $\begin{array}{l}\text { Primer y segun- } \\
\text { do molar prima- } \\
\text { rio }\end{array}$ & 4 & 1,3 \\
\hline $\begin{array}{l}\text { Primer molar } \\
\text { permanente }\end{array}$ & 17 & 5,5 \\
\hline $\begin{array}{l}\text { Incisivo central } \\
\text { permanente }\end{array}$ & 3 & 0,1 \\
\hline $\begin{array}{l}\text { Incisivo lateral } \\
\text { permanente }\end{array}$ & 1 & 0,3 \\
\hline $\begin{array}{l}\text { Segundo molar } \\
\text { primario y prim- } \\
\text { er molar perma- } \\
\text { nente }\end{array}$ & 51 & 16,6 \\
\hline Total & 310 & 100 \\
\hline
\end{tabular}

Tabla 5. Distribución de frecuencia de acuerdo al diente más afectado en niños entre 1 a 6 años que presentaron celulitis facial de origen odontogénico hospitalizados en el Servicio de Infectología Pediátrica del Hospital Universitario de Caracas entre enero de 2006 a diciembre de 2014. 
Los estudios epidemiológicos sobre la prevalencia de la celulitis facial odontogénica en pacientes entre 1 - 6 años son muy pocos, en este sentido y luego de realizar una extensa revisión de la literatura se encontró que en la mayoría de los casos no está claro el diseño metodológico de la investigación, la población, ni el tiempo de estudio, lo que hace más difícil y complicado la realización de esos análisis. ${ }^{8}$

Biederman G y Dodson T, hicieron un reporte epidemiológico de 143 pacientes pediátricos menores de 15 años hospitalizados por celulitis facial de origen odontogénico en Norteamérica en 1994. ${ }^{9}$ Por su parte, Gómez J y Cols. en 1996 en Madrid encontraron una prevalencia de $21 \%$ de celulitis facial de origen odontogénico en niños de entre 5 y 10 años de edad. ${ }^{10}$ Unkel J y Cols. ${ }^{2}$ en un estudio realizado en un Hospital pediátrico de Pittsburg, Estados Unidos en 1997, señalan que 50\% de las afecciones faciales registradas en 100 pacientes pediátricos que fueron admitidos en esa institución, se correspondieron con celulitis facial de origen odontogénico. A diferencia de este estudio en donde la prevalencia fue del 5,27\%, siendo baja en relación con los reportes previamente mencionados. Sin embargo, es importante destacar que en el Servicio en el cual se tomó esta muestra, es altamente especializado (Infectología Pediátrica) y el principal motivo de hospitalización es a causa de enfermedades tropicales como dengue y mal de Chagas, a su vez también hay ingresos por tuberculosis, VIH/SIDA, infecciones respiratorias, entre otras. También es de interés resaltar que esta prevalencia fue tomada a partir de la muestra total de población hospitalizada en este Servicio durante el periodo estudiado, en este sentido al determinar la prevalencia de celulitis facial de origen odontogénico a partir del total de pacientes hospitalizados con diagnóstico de celulitis facial, se obtuvo que la misma era del $42,87 \%$, resultado que indica que de cada 100 niños hospitalizados por celulitis facial, 43 presentan un origen odontogénico.

Al realizar este estudio, se encontró que fueron atendidos un total de 5882 pacientes pediátricos en el Servicio de Infectología Pediátrica del Hospital Universitario de Caracas con edades entre 1 a 6 años en el periodo comprendido entre Enero de 2006 a Diciembre de 2014, de los cuales 310 ni- ños fueron hospitalizados por celulitis facial de origen odontogénico, lo que se corresponde con una prevalencia de 5,27\%, resultado que se acerca bastante a la prevalencia obtenida en el estudio venezolano realizado por Barrios $\mathrm{Z}$ y Carrero T, en 2011 la cual fue de 3,23\%. ${ }^{11}$ Sin embargo, si sólo se considera la prevalencia de celulitis facial en la población estudiada, el origen odontogénico representó un $42,87 \%$ del total.

En relación con la distribución de la población afectada en cuanto al género, de los 310 pacientes atendidos con diagnóstico de celulitis facial de origen odontogénico en esta investigación, 199 pacientes eran de género masculino 64,2\% y 111 pacientes de género femenino (35,8\%). Resultados similares encontraron Gómez J y Cols.en el año 1996 en donde afirman que el 63\% de la población era de género masculino, ${ }^{10}$ Rodríguez $\mathrm{O}$, en el 2001 encontró una prevalencia mayor en el género masculino de 56,37\% , ${ }^{12}$ Barrios Z y Carrero T, en 2011 reportaron que el 56,05\% de la población afectada en su investigación era de género masculino.11 Kim M y Cols.en 2011 afirman que el género masculino tuvo una mayor prevalencia con 52\%. ${ }^{13}$ Grandas, A y Velásquez S en 2012 obtuvieron como resultado que el género masculino fue el más afectado con un 59,04\%, 5 Kara A y Cols. en 2014 hallaron una prevalencia de 58,49\% en el género masculino y $41,51 \%$ para el género femenino.7 Michael J y Hibbert S, en 2014 encontraron que el género masculino fue el más afectado en un $55 \% .{ }^{14}$ Sin embargo estos resultados discrepan de los referidos por Medina J, en el 2012 cuya prevalencia del género femenino fue mayor con un $59 \%$ del total de su muestra de estudio. ${ }^{15}$

Con respecto a la edad, en este estudio se evaluaron niños entre uno y seis años y se encontró que el grupo etario más afectado estaba entre los cuatro y seis años, con una prevalencia de 8,5\% (aproximadamente 9 por cada 100), en comparación con el grupo entre uno y tres años en los que la prevalencia fue representativamente menor de 3,1\% (aproximadamente 3 por cada 100), esto indica que la edad constituye un factor predisponente a la presencia de celulitis facial de origen odontogénico en niños de cuatro a seis años. Estos resultados coinciden con el estudio realizado por Dodson T y Cols. en el año 1989, en 
California, Estados Unidos de Norteamérica, en el cual reportaron que la edad más afectada por celulitis facial fue la de 4 años en el maxilar y 5,56 años en la mandíbula. ${ }^{16}$ Gómez J y Cols. en 1996, encontraron que la edad más afectada era la de 5 años edad ${ }^{10}$. Rodríguez $\mathrm{O}$, en 2001 también reportó una mayor prevalencia en el grupo etario de 2-11 años en un 34\% de su muestra. ${ }^{12}$ Lin $Y$ y Lu P, en 2006 determinaron en su estudio que la edad más afectada estaba comprendida entre el rango de 5,72 $\pm 2,70$ años. ${ }^{17}$ Este estudio también coincide con el trabajo de Thikkurissy, S. y Cols.en Norteamérica, en el año 2010 en el cual se obtuvo una media de edad de $8,3 \pm 3,8$ años de edad afectada por la celulitis facial de origen odontogénico.18 Medina J, en 2012 igualmente encontró que la edad más afectada por esta entidad se encontraba entre los 4 y 6 años de edad ${ }^{15} y$ Grandas A y Velásquez S afirman que hay mayor prevalencia en niños entre 3 a 5 años de edad lo que en su estudio representó el 42,55\%. ${ }^{5}$ Kuo J y Cols.encontraron una media de edad en su estudio de 5,10 $\pm 2,27$ años, ${ }^{19}$ Kara A y Cols. en 2014 determinaron que el rango de edad más afectado con celulitis facial de origen odontogénico estaba entre 7,28 $\pm 3,36$ años de edad. ${ }^{7}$ Por su parte, Michael J y Hebbert S, ${ }^{14}$ en 2014 obtuvieron una media de edad afectada con celulitis facial de origen odontogénico de 6 años.

Con respecto a la distribución de frecuencia según el grupo dental, se encontró que los dientes primarios fueron los más afectados con una prevalencia del $76,8 \%$ con respecto a los dientes permanentes los cuales estuvieron involucrados en un $6,7 \%$ de los pacientes hospitalizados, por su parte, la afectación de dientes primarios y permanentes se observó en el 16,5\% de la población estudiada. Lin Y y Lu P 17 en 2006, Kuo J y Cols. ${ }^{19}$ en 2013 y Kara A y Cols. ${ }^{7}$ en 2014, concluyeron en sus estudios que, la dentición más afectada fue la dentición primaria concordando con la presente investigación. En este estudio se encontró que existe significancia estadística entre la afección de dientes primarios y la presencia de celulitis facial de origen odontogénico.

En esta investigación, el diente más afectado fue el segundo molar primario obteniendo una prevalencia del 49,5\%, seguido del primer molar primario con $22,3 \%$ y del segundo molar primario en conjunto con el primer molar permanente $16,6 \%$, por su parte, el primer molar permanente estuvo afectado de manera aislada únicamente en el 5,5\% de los pacientes, sin embargo, no se encontró relevancia estadísticamente significativa que relacione la celulitis facial de origen odontogénico con el diente más afectado en esta investigación. Estos resultados no se corresponden con el estudio de Barrios $\mathrm{Z}$ y Carrero $\mathrm{T}^{11}$ en el 2011, quienes indican que el diente más comúnmente afectado es el primer molar primario en un 30\% seguido del incisivo central en un $25 \%$. De igual manera, Grandas A y Velásquez $S^{5}$ en el 2012, establecieron que el primer molar primario fue el diente más afectado en un $23 \%$ de los pacientes, Kuo J y Cols. ${ }^{19}$ en el 2013 concluyeron que los dientes más afectados fueron los primeros molares primarios seguidos de los segundos molares primarios y Kara A y Cols. en el 2014 quienes también hallaron mayor prevalencia en el primer molar primario en un $22 \%$.

\section{Conclusiones:}

Para esta muestra, la prevalencia de celulitis facial de origen odontogénico en comparación con otras infecciones que requieren hospitalización fue baja.

El género masculino fue el más afectado y el grupo etario que mayormente implicado fue el comprendido entre los 4 y 6 años de edad.

Los dientes primarios fueron los más afectados y el diente más asociado con esta afección fue el segundo molar primario. 


\section{Referencias bibliográficas.}

1. Lima M. Impacto social de la estrategia para disminuir la incidencia de celulitis facial odontógena. Rev Med Electrón. 2009; 31(5).

2. Unkel J, Mckibben D, Fenton S, Nazif M, Moursi A, Schuit K. Comparison of odontogenic and non odontogenic facial cellulitis in a pediatric hospital population. Pediatric Dent. 1997; 19(8): 476-9.

3. González S, Torales T, Gómez B. Infectología clínica pediátrica DF, México: Mc Graw-Hill Interamericana; 2004.

4. Cores A, Chaviano J, Mazaira L, Atienza L, Salas M. Tratamiento y evolución de la celulitis facial odontógena en edad pediátrica. Gaceta Dental. 2008; 162-181.

5. Grandas A, Velásquez S. Prevalencia de celulitis odontogénica en pacientes de 0-18 años que asistieron a la Fundación HOMI- Hospital de la Misericordida de Bogota entre febrero de 2009- febrero 2011. Acta Odontológica Colombiana. 2012; 2(1): 71-85.

6. Declaración de Helsinki de la Asociación Médica Mundial. In Asociación Médica Mundial; 2013; Fortaleza, Brasil. 64.

7. Kara A, Ozsurekci Y, Tekcicek M, Oncel E, Cengiz A, Karahan S, et al. Length of hospital stay an management of facial cellulitis of odontogenic origin in children. Pediatric Dentistry. 2014; 36(1): 18-22.

8. Wang J, Ahani A, Pogrel M. A five-year retrospective study of odontogenic maxillofacial infections in a large urban public hospital. Int J Oral Maxillofac Surg. 2005; 34(6): 646-9.

9. Biederman G, Dodson T. Epidemiologic review of facial infections in hospitalized pediatric patients. J Oral Maxillofac Surg. 1994; 52(10): 1042-5.

10. Gómez J, Navarro M, García-Mon F, Arguez G, Casanova A. Celulitis orbitaria y periorbitaria en la infancia. Revisión de 116 casos. Anales Españoles de Pediatría. 1996; 44(1): 29-34.

11. Barrios Z, Carrero T. Prevalencia de infecciones de origen pulpar en los niños atendidos en el Servicio de Odontopediatría del Instituto Autónomo Hospital Universitario de los Andes, Mérida, Venezuela. Revista Odontológica de los Andes. 2011; 6(1): 42-51.

12. Rodríguez O. Diagnóstico y tratamiento de la celulitis facial odontógena. Acta Odontol Venez. 2001; 39(3).

13. Kim M, Nalliah R, Lee M, Allareddy V. Factors associated with length of stay and hospital charges for patients hospitalized with mouth cellulitis. Oral Surg Oral Med Oral Pathol Oral Radiol Endod. 2011; 113(1): 21-28.

14. Michael J, Hibbert S. Presentation and management of facial swellings of odontogenic origin in children. Eur Arch Paediatr Dent. 2014; 15(4): 259-68.

15. Medina J. Celulitis facial odontogénica en pacientes hospitalizados en un hospital especializado en pediatría. Dermatol Perú. 2012; 23(2): 73-7.

16. Dodson T, Perrott D, Kaban L. Pediatric maxillofacial infections: a retrospective study of 113 patients. J Oral Maxillofac Surg. 1989; 47(4): 327-30.

17. Lin Y, Lu P. Retrospective study of pediatric facial cellulitis of odontogenic origin. Pediatr Infect Dis J. 2006; $25(4)$ : 339-42.

18. Thikkurissy S, Rawlins J, Kumar A, Evans E, Casamassimo P. Rapid treatment reduces hospitalization for pediatric patients with odontogenic-based cellulitis. Am J Emerg Med. 2010; 28(6): 668-72.

19. Kuo J, Lin Y, Lin Y. Odontogenic cellulitis in children requiring hospitalization. J Dent Sci. 2013; 8(2): 129-32.

Recibido: 25-01-2016

Aceptado: 10-03-2016

Correspondencia: Gabriel Zambrano gazblondell@gmail.com 